Pacific Journal of Mathematics

DISTRIBUTION OF ZEROS OF SOLUTIONS OF A FOURTH 


\section{DISTRIBUTION OF ZEROS OF SOLUTIONS OF A FOURTH ORDER DIFFERENTIAL EQUATION}

\section{A. C. Peterson}

The primary concern of this paper is to study the distribution of zeros of solutions, that have at least four zeros, two or more of which are distinct zeros, of the canonical fourth order equation

$\left(E_{4}\right)$

$$
L_{4}[y]=\left(r_{3} L_{3}[y]\right)^{\prime}+q_{3} r_{2} L_{2}[y]+q_{4} y=0,
$$

where $r_{i}(x)>0, r_{i}(x), q_{j}(x) \in C[a, \infty), i=1,2,3, j=1,2,3,4$, which was introduced by Barrett.

The canonical second order equation

$$
L_{2}[y]=\left(r_{1} y^{\prime}\right)^{\prime}+q_{1} y=0,
$$

where $r_{1}(x)>0, r_{1}(x), q_{1}(x) \in C[a, \infty)$, has been studied extensively. The canonical third order linear differential equation

$$
L_{3}[y]=\left(r_{2} L_{2}[y]\right)^{\prime}+q_{2}\left(r_{1} y^{\prime}\right)=0,
$$

where $r_{i}(x)>0, r_{i}(x), q_{i}(x) \in C[a, \infty), i=1,2$, which was introduced by Barrett, is a generalization of the second order equation $\left(E_{2}\right)$.

Dolan studied the distribution of zeros of extremal solutions of $\left(E_{3}\right)$ for the first conjugate point $\eta_{1}(t)$. In paragraph 2 the same study is made for the equation $\left(E_{4}\right)$ and many of Dolan's ideas and techniques are used there. The results in paragraph 2 substantially complete the investigation begun in a paper by Aliev. Aliev defined and investigated the numbers $r_{i j k}(t)$ and $r_{1111}(t)$, which are extensions of the two-point nonoscillation numbers $r_{i j}(t)$ of Azbelev and Caljuk. Several of his results were reported in sources, which did not include the proofs, and these proofs were unavailable to the author, e.g.,

$$
r_{31}(t) \geqq r_{211}(t) \geqq \min \left[r_{22}(t), r_{31}(t)\right] .
$$

Aliev also proved that

$$
r_{1111}(t)=\min \left[r_{121}(t), r_{112}(t)\right],
$$

and purported to prove that $r_{1111}(t)=\min \left[r_{211}(t), r_{112}(t)\right]$ but his proof is incorrect and this remains an open question. In paragraph 3 these results of Aliev are reproved and a much more complete picture is presented in the ordering of the numbers $\eta_{1}(t), r_{i j}(t), r_{i j k}(t)$, and $r_{1111}(t)$. The main results of this paper appear in paragraph 3 . 
1. Introduction. We will be concerned with the fourth order quasi differential equation

$$
L_{4}[y]=\left(D_{3} y\right)^{\prime}+q_{3} D_{2} y+q_{4} y=0,
$$

where

$$
D_{1} y=r_{1} y^{\prime}, D_{2} y=r_{2} L_{2}[y], D_{3} y=r_{3} L_{3}[y] .
$$

For a discussion of the basic properties of $\left(E_{4}\right)$ see Chapter III of [6]. An adjoint differential equation to $\left(E_{4}\right)$ is

$$
L_{4}^{+}[y]=\left(D_{3}^{+} y\right)^{\prime}+q_{1} D_{2}^{+} y+q_{4} y=0,
$$

where $D_{i}^{+} y$ is obtained from $D_{i} y, i=1,2,3$, by interchanging $r_{1}$ with $r_{3}$, and $q_{1}$ with $q_{3}$. Note then that equation $\left(E_{4}^{+}\right)$is obtained from $\left(E_{4}\right)$ by interchanging $r_{1}$ with $r_{3}$, and $q_{1}$ with $q_{3}$. If $\left(E_{4}\right)$ and $\left(E_{4}^{+}\right)$are equivalent, then $L_{4}$ (and the corresponding equation $\left(E_{4}\right)$ ) is said to be self adjoint; e.g., if $r_{1}(x) \equiv r_{3}(x)$ and $q_{1}(x) \equiv q_{3}(x)$ in equation $\left(E_{4}\right)$, then we have a canonical form for the self adjoint equation of order four [6], i.e.,

$$
\begin{aligned}
L_{4}^{*}[y]= & \left\{r_{1}\left[\left(r_{2}\left[\left(r_{1} y^{\prime}\right)^{\prime}+q_{1} y\right]\right)^{\prime}+q_{2} r_{1} y^{\prime}\right]\right\}^{\prime} \\
& +q_{1} r_{2}\left[\left(r_{1} y^{\prime}\right)^{\prime}+q_{1} y\right]+q_{4} y=0 .
\end{aligned}
$$

A fundamental set of solutions $u_{j}(x, t)$ of $\left(E_{4}\right)$ for $t \in[a, \infty)$ is defined by

$$
D_{i} u_{j}(t, t)=\delta_{i j}, i=0,1,2,3, j=0,1,2,3 .
$$

Similarly, a fundamental set of solutions $u_{j}^{+}(x, t), j=0,1,2,3$, is defined for the adjoint equation $\left(E_{4}^{+}\right)$. We call $u_{3}(x, t)$ the first principal solution of $\left(E_{4}\right)$. Leighton and Nehari [10] made use of the identity

$$
u_{3}(s, t)=-u_{3}^{+}(t, s) .
$$

which is a special case of the following theorem which follows from the Lagrange Identity [6] for $\left(E_{4}\right)$.

Theorem 1.1. For $\alpha, \beta=0,1,2,3$ and $s, t \in[a, \infty)$

$$
D_{\alpha} u_{\beta}(s, t)=(-1)^{\alpha+\beta} D_{3-\beta}^{+} u_{3-\alpha}^{+}(t, s) \text {. }
$$

The derivation of equations (1.1) is similar to that given by Dolan [7] for a similar set of formulas for the equation $\left(E_{3}\right)$.

Instead of the usual Wronskians, $\operatorname{det}\left(y_{i}^{(j)}\right)$, involving pure derivatives we introduce the more convenient generalized Wronskians, 


$$
\begin{array}{ll}
W\left[y_{1}, \cdots, y_{n}\right]=\operatorname{det}\left(D_{i} y_{j}\right), & i=0,1, \cdots, n-1 \\
& j=1,2, \cdots, n \\
& n=1,2,3,4 \\
W^{+}\left[y_{1}, \cdots, y_{n}\right]=\operatorname{det}\left(D_{i}^{+} y_{j}\right), &
\end{array}
$$

which are given in terms of quasi derivatives $\left(D_{0} y=D_{0}^{+} y=y\right)$. When we speak of a zero of a solution $y(x)$ of $\left(E_{4}\right)$ of order $k$ at we mean $D_{i} y(t)=0, i=0, \cdots, k-1, k=1,2,3,4$. If $\left(E_{4}\right)$ is disconjugate on $[t, \infty)$ we write $\eta_{1}(t)=\infty$. Otherwise $\eta_{1}(t)$ will denote the first conjugate point of $t$. For properties of $\eta_{1}(t)$ see [9], [12]. A nontrivial solution of $\left(E_{4}\right)$ having four zeros on $\left[t, \eta_{1}(t)\right]$ is called an extremal solution of $\left(E_{4}\right)$ for $\eta_{1}(t)$. A nontrivial solution $y$ of $\left(E_{4}\right)$ is said to have an $i_{0}-i_{1}-\cdots-i_{\nu}\left(\nu=1,2,3 ; i_{k}=1,2,3\right)$ distribution of zeros on $[t, b] \subset[a, \infty)$ provided $y$ has a zero at $t_{k}$ of order at least $i_{k}$ where $t \leqq t_{0}<t_{1}<\cdots<t_{\nu} \leqq b$. We now can introduce the following concepts, introduced by Dolan [7] for $\left(E_{3}\right)$.

Definition 1.1. For $t \in[a, \infty)$, the number $z_{i_{0} i_{1} \cdots i_{\nu}}(t)$ is the infimum of the set of numbers $b>t$ such that there is a nontrivial solution $y$ of $\left(E_{4}\right)$ having an $i_{0}-i_{1}-\cdots-i_{\nu}$ distribution of zeros on $[t, b]$ and a zero of order at least $i_{0}$ at $t_{0}$. By $z_{i_{0} i_{1} \cdots i_{\nu}}(t)=\infty$ we mean there is no such distribution of zeros on $[t, \infty)$.

Definition 1.2. For $t \in[a, \infty)$, the number $r_{i_{0} i_{1} \cdots i_{\nu}}(t)$ is the infimum of the set of numbers $b>t$ such that there is a nontrivial solution $y$ of $\left(E_{4}\right)$ having an $i_{0}-i_{1}-\cdots-i_{\nu}$ distribution of zeros on $[t, b]$, by $r_{i_{0} i_{1} \cdots i_{\nu}}(t)=\infty$ we mean there is no such distribution of zeros on $[t, \infty)$. The numbers $z_{i_{0} i_{1} \cdots i_{\nu}}^{+}(t)$ and $r_{i_{0} i_{1} \cdots i_{\nu}}^{+}(t)$ are defined similarly for the adjoint equation $\left(E_{4}^{+}\right)$.

If $z_{i j}(t)<\infty\left\{r_{i j}(t)<\infty\right\}$, then the word "infimum" in Definition $1.1\{1.2\}$ can be replaced by "minimum". However, if $\nu>1$ in Definitions 1.1 and 1.2 then you cannot in general do this (see paragraphs 2 and 3). There is a close relation between zeros of solutions and uniqueness problems, for example if $\alpha, \beta, \gamma$ are numbers such that $t \leqq$ $\alpha<\beta<\gamma<r_{121}(t) \leqq \infty$, then there is a unique solution of $\left(E_{4}\right)$ satisfying

$$
y(\alpha)=A, y(\beta)=B, D_{1} y(\beta)=C, y(\gamma)=D
$$

where $A, B, C, D$ are constants. Corresponding statements hold for the other numbers in Definitions 1.1 and 1.2 (see [2] or [4]). For known properties of these numbers see [1]-[6].

It is convenient to use further notation introduced by Dolan [7]. 
DEFINITION 1.3. If $z_{i j}(t)<\infty\left\{r_{i j}(t)<\infty\right\}$ and $i+j=4$, then

$$
\begin{aligned}
Z_{1}(t) & =\max \left\{z_{31}(t), z_{22}(t), z_{13}(t)\right\} \\
\left\{R_{1}(t)\right. & \left.=\max \left\{r_{31}(t), r_{22}(t), r_{13}(t)\right\}\right\} .
\end{aligned}
$$

By $Z_{1}(t)=\infty\left\{R_{1}(t)=\infty\right\}$ we mean at least one of the $z_{i j}(t)\left\{r_{i j}(t)\right\}$, $i+j=4$, is infinite.

The next lemma appears and is used quite often ([4], [10], [12]) and is stated here in terms of the equation $\left(E_{4}\right)$.

LEMMA 1.2. Let $u(x)$ be a nontrivial solution of $\left(E_{4}\right)$ with a zero at $t_{1} \in[a, \infty)$ of order $n \geqq 1$ and a zero at $t_{2} \in\left(t_{1}, \infty\right)$ of order $m \geqq 1$ and $u(x)>0$ on $\left(t_{1}, t_{2}\right)$. Let $v(x)$ be a solution of $\left(E_{4}\right)$ such that $v(x)>0$ on $\left(t_{1}, t_{2}\right)$ and $v(x)$ does not have a zero of order $\geqq n$ at $t_{1}$ and does not have a zero of order $\geqq m$ at $t_{2}$. Then there are constants $c_{1}, c_{2}$ such that $c_{1} c_{2}<0$ and $z(x)=c_{1} u(x)+c_{2} v(x)$ is a solution of $\left(E_{4}\right)$ with a zero of order two in $\left(t_{1}, t_{2}\right)$.

\section{The distribution of zeros of extremal solutions.}

I. Distribution of zeros when $\eta_{1}(t)<Z_{1}(t)$. In this part of paragraph 2 we study the distribution of zeros of extremal solutions of $\left(E_{4}\right)$ for $\eta_{1}(t)$ when $\eta_{1}(t)<Z_{1}(t)$. Since $\eta_{1}(t)<z_{i j}(t)$ if and only if $\eta_{1}(t)<r_{i j}(t), \eta_{1}(t)<Z_{1}(t)$ if and only if $\eta_{1}(t)<R_{1}(t)$. Thus the theorems in this chapter are true when the assumption $\eta_{1}(t)<Z_{1}(t)$ is replaced by $\eta_{1}(t)<R_{1}(t)$.

Hartman [8] proves for an $n^{\text {th }}$ order linear homogeneous differential equation that no nontrivial solution has $n$ zeros, counting multiplicities, on an open interval $(\alpha, \beta)$ if and only if no nontrivial solution has $n$ distinct zeros on the open interval $(\alpha, \beta)$. Hartman raised the question as to whether you could or could not replace "open interval $(\alpha, \beta)$ " by "closed interval $[\alpha, \beta]$ " in the preceding statement. The next lemma shows that you can not replace "open interval $(\alpha, \beta)$ " by "closed interval $[\alpha, \beta]$ ". Dolan [7] has established a similar theorem for a third order differential equation. A similar result would hold for an $n^{\text {th }}$ order linear homogeneous differential equation.

Lemma 2.1. If $\eta_{1}(t)<Z_{1}(t) \leqq \infty$ for $t \in[a, \infty)$, then no extremal solution of $\left(E_{4}\right)$ for $\eta_{1}(t)$ has four distinct zeros on $\left[t, \eta_{1}(t)\right]$.

Proof. Assume $u(x)$ is an extremal solution of $\left(E_{4}\right)$ for $\eta_{1}(t)$ with four distinct zeros on $\left[t, \eta_{1}(t)\right]$. Since $\eta_{1}(t)$ is a strictly increasing function $u(x)$ has exactly four simple zeros on $\left[t, \eta_{1}(t)\right]$ and $u(t)=$ 
$u\left(\eta_{1}(t)\right)=0$. Since $\eta_{1}(t)<Z_{1}(t) \leqq \infty$, there are three possibilities.

Case 1. $\eta_{1}(t)<z_{13}(t) \leqq \infty$.

For $\varepsilon>0$, sufficiently small, let $\left\{u_{\varepsilon}(x)\right\}$ be a set of nontrivial solutions of $\left(E_{4}\right)$ satisfying

$$
\begin{gathered}
u_{\varepsilon}(t)=0=u_{\varepsilon}\left(\eta_{1}(t)-\varepsilon\right) \\
D_{1} u_{\varepsilon}\left(\eta_{1}(t)-\varepsilon\right)=D_{1} u\left(\eta_{1}(t)\right) \neq 0 \\
D_{2} u_{\varepsilon}\left(\eta_{1}(t)-\varepsilon\right)=D_{2} u\left(\eta_{1}(t)\right) .
\end{gathered}
$$

Since $u_{3}\left(t, \eta_{1}(t)\right) \neq 0$, it is easy to see that as $\varepsilon \rightarrow 0$

$$
u_{\varepsilon}(x) \rightarrow u(x) \text { uniformly for } x \in\left[t, \eta_{1}(t)\right] .
$$

Since the zeros of $u(x)$ in $\left(t, \eta_{1}(t)\right)$ are simple, there is an $\varepsilon_{0}>0$ such that $u_{\varepsilon_{0}}(x)$ is a solution of $\left(E_{4}\right)$ with four zeros on $\left[t, \eta_{1}(t)-\varepsilon_{0}\right]$. This contradicts the definition of $\eta_{1}(t)$.

Case 2. $\eta_{1}(t)<z_{22}(t) \leqq \infty$.

For $\varepsilon>0$, sufficiently small, let $\left\{u_{\varepsilon}(x)\right\}$ be a set of nontrivial solutions of $\left(E_{4}\right)$ satisfying

$$
\begin{aligned}
& u_{\varepsilon}(t)=0=u_{\varepsilon}\left(\eta_{1}(t)-\varepsilon\right) \\
& D_{1} u_{\varepsilon}(t)=D_{1} u(t) \neq 0 \\
& D_{1} u_{\varepsilon}\left(\eta_{1}(t)-\varepsilon\right)=D_{1} u\left(\eta_{1}(t)\right) .
\end{aligned}
$$

Using the fact that $W\left[u_{2}\left(\eta_{1}(t), t\right), u_{3}\left(\eta_{1}(t), t\right)\right] \neq 0$, we proceed as in Case 1 to obtain a contradiction.

Case 3. $\eta_{1}(t)<z_{31}(t) \leqq \infty$.

A similar argument disposes of this case.

Aliev [5] proved for the classical fourth order equation that if $r_{13}(t)<\min \left[r_{31}(t), r_{22}(t)\right], r_{31}(t)<\min \left[r_{13}(t), r_{22}(t)\right]$, or $r_{22}(t)<\min \left[r_{13}(t)\right.$, $\left.r_{31}(t)\right]$, then no nontrivial solution of $l_{4}[y]=0$ has four distinct zeros on $\left[t, \eta_{1}(t)\right]$. Lemma 2.1 generalizes his Theorems 1, 3, and 5. Another way in which Lemma 2.1 can be generalized, which is also similar to a result of M. Dolan [7] for $\left(E_{3}\right)$, is the following theorem.

TheOREm 2.2. If, for $t \in[a, \infty), \eta_{n}(t)<\infty$ and if one of the inequalities $u_{3}\left(\eta_{n}(t), t\right) \neq 0, W\left[u_{2}\left(\eta_{n}(t), t\right), u_{3}\left(\eta_{n}(t), t\right)\right] \neq 0, u_{3}\left(t, \eta_{n}(t)\right) \neq 0$ holds, then no extremal solution of $\left(E_{4}\right)$ for $\eta_{n}(t)$ has all simple zeros on $\left[t, \eta_{n}(t)\right]$.

The proof of Theorem 2.2 is similar to the proof of Lemma 2.1. 
Obviously a theorem like this can be stated for a more general linear $n^{\text {th }}$ order equation.

Lemma 2.3. If, for $t \in[a, \infty), \eta_{1}(t)<Z_{1}(t) \leqq \infty$, then no extremal solution of $\left(E_{4}\right)$ for $\eta_{1}(t)$ has a $1-2-1$ distribution of zeros on $\left[t, \eta_{1}(t)\right]$.

Proof. Assume $u(x)$ is a nontrivial solution of $\left(E_{4}\right)$ with a $1-2-1$ distribution of zeros on $\left[t, \eta_{1}(t)\right]$, then $u(x)$ has a simple zero at $t$, a simple zero at $\eta_{1}(t)$, a double zero at some point $\tau \in\left(t, \eta_{1}(t)\right)$, and we can assume $u(x)>0$ for $x \in(t, \tau) \cup\left(\tau, \eta_{1}(t)\right)$. It follows that there is a nontriviál linear combination of $u(x)$ and $u_{3}(x, t)$ with four distinct zeros on $\left[t, \eta_{1}(t)\right]$ which contradicts Lemma 2.1.

Lemma 2.4. If, for $t \in[a, \infty), \eta_{1}(t)<Z_{1}(t) \leqq \infty$, then no extremal solution of $\left(E_{4}\right)$ for $\eta_{1}(t)$ has a 2-1-2 distribution of zeros on $\left[t, \eta_{1}(t)\right]$.

Proof. Let $u(x)$ be a nontrivial solution of $\left(E_{4}\right)$ with a $2-1-2$ distribution of zeros on $\left[t, \eta_{1}(t)\right]$, then $u(x)$ has a double zero at $t$, a double zero at $\eta_{1}(t)$, a simple zero at some point $\tau \in\left(t, \eta_{1}(t)\right)$, and no other zeros on $\left[t, \eta_{1}(t)\right]$. It is easy to see that $u_{3}(x, t)$ does not have a multiple zero at $\eta_{1}(t)$, and so we can apply Lemma 1.2 to $u(x)$ and $u_{3}(x, t)$ to get a contradiction.

It follows as a corollary to Lemma 2.4 that if $\eta_{1}(t)<\infty$, then there is an extremal solution of $\left(E_{4}\right)$ for $\eta_{1}(t)$ which has a sum of at least four zeros at $t$ and $\eta_{1}(t)$ and is nonzero in $\left(t, \eta_{1}(t)\right)$. This is a special case of the general $n^{\text {th }}$ order results of Sherman [12] and Hinton [9].

It is evident that if $z_{31}(t)=z_{22}(t)<z_{13}(t)\left\{z_{13}(t)=z_{22}(t)<z_{31}(t)\right\}$ and if $\eta_{1}(t)<z_{32}(t)\left\{\eta_{1}(t)<z_{23}(t)\right\}$, then there is an extremal solution of $\left(E_{4}\right)$ for $\eta_{1}(t)$ with a $2-1-1\{1-1-2\}$ distribution of zeros on $\left[t, \eta_{1}(t)\right]$. Hence, the condition $\eta_{1}(t)<Z_{1}(t) \leqq \infty$ is not enough to ensure that no extremal solution of $\left(E_{4}\right)$ for $\eta_{1}(t)$ has a $2-1-1$ or a $1-1-2$ distribution of zeros on $\left[t, \eta_{1}(t)\right]$. Lemmas 2.5 and 2.6 give partial answers to this quandary.

LEMMA 2.5. If, for

$$
t \in[a, \infty), \eta_{1}(t)<\max \left[z_{22}(t), z_{13}(t)\right]\left\{\eta_{1}(t)<\max \left[z_{22}(t), z_{31}(t)\right]\right\},
$$

then no extremal solution of $\left(E_{4}\right)$ for $\eta_{1}(t)$ has a $1-1-2\{2-1-1\}$ distribution of zeros on $\left[t, \eta_{1}(t)\right]$.

Proof. Assume $\eta_{1}(t)<\max \left[z_{22}(t), z_{13}(t)\right]$ and $u(x)$ is a nontrivial 
solution of $\left(E_{4}\right)$ with a $1-1-2$ distribution of zeros on $\left[t, \eta_{1}(t)\right]$, then $u(x)$ has a simple zero at $t$, a double zero at $\eta_{1}(t)$, a simple zero at some point $\tau \in\left(t, \eta_{1}(t)\right)$, and no other zeros on $\left[t, \eta_{1}(t)\right]$. If $z_{13}(t)>\eta_{1}(t)$ we get a contradiction by applying Lemma 1.2 to $u_{3}(x, t)$ and $u(x)$. Otherwise there is a nontrivial linear combination of $u(x)$ and $u_{3}\left(x, \eta_{1}(t)\right)$ with a double zero at $t$ and at $\eta_{1}(t)$ which contradicts $z_{22}(t)>\eta_{1}(t)$. The proof of the second half of this theorem is similar.

ExAMPLE 2.1. $y^{i v}-y^{\prime}=0$. Aliev [4] noted that $\eta_{1}(t)=r_{13}(t) \approx$ $t+5.9, r_{31}(t)=r_{22}(t)=\infty$. It follows from Lemma 2.5 that no nontrivial solution of $y^{i v}-y^{\prime}=0$ has a $2-1-1$ distribution of zeros on $\left[t, \eta_{1}(t)\right]$.

EXAMPLE 2.2. $y^{i v}+y^{\prime}=0$. Since, for $t$ real, $\eta_{1}(t)=r_{31}(t) \approx t+5.9$ and $r_{13}(t)=r_{22}(t)=\infty$, we have by Lemma 2.5 that no nontrivial solution has a $1-1-2$ distribution of zeros on $\left[t, \eta_{1}(t)\right]$.

LEMma 2.6. If, for $t \in[a, \infty)$, equation $\left(E_{4}\right)$ is self adjoint and if $\eta_{1}(t)<Z_{1}(t) \leqq \infty$, then no extremal solution of $\left(E_{4}\right)$ for $\eta_{1}(t)$ has a $1-1-2$ or $2-1-1$ distribution of zeros on $\left[t, \eta_{1}(t)\right]$.

Proof. Assume $u(x)$ is a nontrivial solution of $\left(E_{4}\right)$ with a $2-1-1$ distribution of zeros on $\left[t, \eta_{1}(t)\right]$, then $u(x)$ has a double zero at $t$, a simple zero at $\eta_{1}(t)$, a simple zero at some $\tau \in\left(t, \eta_{1}(t)\right)$, and no other zeros on $\left[t, \eta_{1}(t)\right]$. Since $\left(E_{4}\right)$ is self adjoint $z_{13}(t)=z_{31}(t)$ and $\eta_{1}(t)<$ $Z_{1}(t)$ is possible in two ways.

If $\eta_{1}(t)=z_{22}(t)<z_{31}(t)=z_{13}(t)$, then there is a nontrivial solution $v(x)$ of $\left(E_{4}\right)$ with a double zero at $t$ and a double zero at $\eta_{1}(t)$. It follows that there is a nontrivial linear combination of $u(x)$ and $v(x)$ with a triple zero at $t$ and a zero at $\eta_{1}(t)$. This contradicts the inequality $\eta_{1}(t)<z_{31}(t)$.

If $\eta_{1}(t)=z_{13}(t)=z_{31}(t)<z_{22}(t)$, then for $\varepsilon>0$, sufficiently small, let $\left\{u_{\varepsilon}(x)\right\}$ be a set of nontrivial solutions of $\left(E_{4}\right)$ satisfying

$$
\begin{gathered}
u_{\varepsilon}(t)=D_{1} u_{\varepsilon}(t)=0 \\
u_{\varepsilon}\left(\eta_{1}(t)-\varepsilon\right)=0 \\
D_{1} u_{\varepsilon}\left(\eta_{1}(t)-\varepsilon\right)=D_{1} u\left(\eta_{1}(t)\right) \neq 0 .
\end{gathered}
$$

Since $\eta_{1}(t)<z_{22}(t), W\left[u_{2}\left(\eta_{1}(t), t\right), u_{3}\left(\eta_{1}(t), t\right)\right] \neq 0$ and it follows that as $\varepsilon \rightarrow 0$

$$
u_{s}(x) \rightarrow u(x) \text { uniformly for } x \in\left[t, \eta_{1}(t)\right] .
$$

Since $\tau$ is a simple zero of $u(x)$, there is an $\varepsilon_{0}>0$ such that $u_{\varepsilon_{0}}(x)$ is a nontrivial solution of $\left(E_{4}\right)$ with a $2-1-1$ distribution of zeros on 
$\left[t, \eta_{1}(t)-\varepsilon_{0}\right]$. This contradicts the definition of $\eta_{1}(t)$. The other half of this theorem is proved similarly.

CoRollary 2.7. If, for $t \in[a, \infty), \eta_{1}(t)<\min \left[r_{13}(t), r_{22}(t)\right], \eta_{1}(t)<$ $\min \left[r_{31}(t), r_{22}(t)\right]$ or $\eta_{1}(t)<\min \left[r_{13}(t), r_{31}(t)\right]$, then no extremal solution of $\left(E_{4}\right)$ for $\eta_{1}(t)$ has three distinct zeros on $\left[t, \eta_{1}(t)\right]$ of which one is at least a double zero.

Proof. This corollary follows from Lemmas 2.3 and 2.4, and a closer look at the proof of Lemma 2.6.

Corollary 2.7 gives Theorems 2, 4, and 5 of Aliev [5] for the more general equation $\left(E_{4}\right)$. Lemmas $2.3-2.6$ are generalizations of these theorems of Aliev [5] for the equation $\left(E_{4}\right)$.

The next theorem characterizes extremal solutions of $\left(E_{4}\right)$ for $\eta_{1}(t)$ when $\left(E_{4}\right)$ is self adjoint and $\eta_{1}(t)<Z_{1}(t) \leqq \infty$. In particular, it shows that the extremal solutions guaranteed by Sherman [12] are the only ones in certain cases. It follows easily from Lemmas 2.1, 2.3-2.6.

THEOREM 2.8. If equation $\left(E_{4}\right)$ is self adjoint and $\eta_{1}(t)<Z_{1}(t) \leqq$ $\infty$, then no extremal solution of $\left(E_{4}\right)$ for $\eta_{1}(t)$ has a zero on $\left(t, \eta_{1}(t)\right)$.

In the special case of Theorem 2.8, when equation $\left(E_{4}\right)$ is self adjoint with $\eta_{1}(t)<z_{22}(t)$, it is interesting to note that, even though no nontrivial solution of $\left(E_{4}\right)$ with four zeros on $\left[t, \eta_{1}(t)\right]$ has a zero in $\left(t, \eta_{1}(t)\right)$, given any $\varepsilon>0$ there is a nontrivial solution to $\left(E_{4}\right)$ with a $2-1-1,1-2-1$, and $1-1-2$ distribution of zeros on $\left[t, \eta_{1}(t)+\varepsilon\right]$ the first zero being at $t$. This is the essence of part (i) of Corollary 3.9 in paragraph 3 . Theorems $3.4,3.6$, and 3.7 are generalizations of part (i) of Corollary 3.9. In the other case of Theorem 2.8, when equation $\left(E_{4}\right)$ is self adjoint with $\eta_{1}(t)<z_{31}(t)$, it is interesting to note that, even through no nontrivial solution of $\left(E_{4}\right)$ with four zeros on $\left[t, \eta_{1}(t)\right]$ has a zero in $\left(t, \eta_{1}(t)\right)$, given any $\varepsilon>0$ there is a solution with a $2-1-1$ and $1-1-2$ distribution of zeros on $\left[t, \eta_{1}(t)+\varepsilon\right]$. This is the essence of part (ii) of Corollary 3.9 in paragraph 3, where we establish a generalization (Theorem 3.8) of this result.

II. Distribution of zeros when $\eta_{1}(t)=Z_{1}(t)$. In this part of paragraph 2 we study the distribution of zeros of extremal solutions of $\left(E_{4}\right)$ for $\eta_{1}(t)$ when $\eta_{1}(t)=Z_{1}(t)$, i.e., when

$$
\eta_{1}(t)=z_{13}(t)=z_{22}(t)=z_{31}(t)<\infty .
$$

The following lemma is very useful. 
Lemma 2.9. If $v_{1}(x), v_{2}(x)$, and $v_{3}(x)$ are three linearly independent solutions of $\left(E_{4}\right)$ with zeros at $t$ and $\eta_{1}(t)$, then there is an extremal solution of $\left(E_{4}\right)$ for $\eta_{1}(t)$ with four distinct zeros on $\left[t, \eta_{1}(t)\right]$.

Proof. Since $v_{1}, v_{2}$, and $v_{3}$ are three linearly independent solutions of $\left(E_{4}\right), w^{+}(x)=W\left[v_{1}, v_{2}, v_{3}\right]$ is a nontrivial solution of the adjoint equation $\left(E_{4}^{+}\right)$[6] and it follows by the formulas (3.19) in [6] that $w^{+}(x)$ has a triple zero at $t$ and at $\eta_{1}(t)$. Let $\alpha, \beta$ be distinct numbers in $\left(t, \eta_{1}(t)\right)$, then $w(x)=W^{+}\left[w^{+}(x), u_{3}^{+}(x, \alpha), u_{3}^{+}(x, \beta)\right]$ is a nontrivial solution of $\left(E_{4}\right)$ which has zeros at $t, \alpha, \beta$, and $\eta_{1}(t)$.

We can now easily prove the following theorem.

TheOREM 2.10. If, for $t \in[a, \infty), \eta_{1}(t)=Z_{1}(t)<\infty$ and

$$
\eta_{1}(t)<\min \left\{z_{23}(t), z_{32}(t)\right\},
$$

then for each of the distributions $1-1-1-1,1-1-2,1-2-1$, and 2-1-1 of zeros on $\left[t, \eta_{1}(t)\right]$ there is an extremal solution of $\left(E_{4}\right)$ for $\eta_{1}(t)$. In fact for any $\alpha, \beta, t \leqq \alpha \leqq \beta \leqq \eta_{1}(t)$ there is a nontrivial solution of $\left(E_{4}\right)$ satisfying $y(t)=y(\alpha)=y(\beta)=y\left(\eta_{1}(t)\right)=0$. (By $t=\alpha<\beta<\eta_{1}(t)$ is meant the boundary conditions $y(t)=D_{1} y(t)=$ $y(\beta)=y\left(\eta_{1}(t)\right)=0$, etc.).

Proof. Let $u(x)$ be a nontrivial solution of $\left(E_{4}\right)$ which has exactly a double zero at $t$ and at $\eta_{1}(t)$. Since $\eta_{1}(t)<\min \left\{z_{23}(t), z_{32}(t)\right\}$ it follows that $u_{3}(x, t), u_{3}\left(x, \eta_{1}(t)\right)$, and $u(x)$ are three linearly independent solutions of $\left(E_{4}\right)$ with zeros at $t$ and $\eta_{1}(t)$. It follows from the proof of Lemma 2.9 that for any $\alpha, \beta, t \leqq \alpha<\beta<\eta_{1}(t)$, there is a nontrivial solution of $\left(E_{4}\right)$ satisfying $y(t)=y(\alpha)=y(\beta)=y\left(\eta_{1}(t)\right)=0$.

If $t<\alpha=\beta<\eta_{1}(t)$ then let $\left\{a_{i}\right\}$ and $\left\{b_{i}\right\}$ be sequences of numbers in $(t, \alpha)$ and $\left(\alpha, \eta_{1}(t)\right)$ respectively such that $\lim _{i \rightarrow \infty} a_{i}=\lim _{i \rightarrow \infty} b_{i}=\alpha$ and let $v_{i}(x)$ be a solution of $\left(E_{4}\right)$ satisfying

$$
v_{i}(t)=v_{i}\left(a_{i}\right)=v_{i}\left(b_{i}\right)=v_{i}\left(\eta_{1}(t)\right)=0 .
$$

It follows by Ascoli's theorem that there is a nontrivial solution of $\left(E_{4}\right)$ satisfying $y(t)=y(\alpha)=D_{1} y(\alpha)=y\left(\eta_{1}(t)\right)=0$. The other parts of this theorem are proved similarly.

The equation [6]

$$
y^{i v}+10 y^{\prime \prime}+9 y=0, t \in[a, \infty),
$$

is self adjoint with $\eta_{1}(t)=z_{33}(t)=t+\pi$. Let $\alpha, \beta \in\left(t, \eta_{1}(t)\right)$, then since $y_{1}(x)=\cos ^{2}(x+\pi / 2-\alpha) \sin (x-t), y_{2}(x)=\sin ^{2}(x-t) \cos (x+\pi / 2-\alpha)$, and $y_{3}(x)=\sin (x-t) \sin (x-\alpha) \sin (x-\beta)$ are solutions of 


$$
y^{i v}+10 y^{\prime \prime}+9 y=0,
$$

there is for each of the distributions $1-2-1,2-1-1,1-1-2$, and $1-1-1-1$ of zeros on $\left[t, \eta_{1}(t)\right]$ an extremal solution of $y^{i v}+10 y^{\prime \prime}+9 y=0$ for $\eta_{1}(t)$. This example suggests the next theorem.

Theorem 2.11. If, for $t \in[a, \infty)$, equation $\left(E_{4}\right)$ is self adjoint with $\eta_{1}(t)=z_{33}(t)$, then the assertions in Theorem 2.10 hold.

Proof. Since $\eta_{1}(t)=z_{33}(t), u_{3}(x, t)$ has a triple zero at $t$ and $\eta_{1}(t)$. Let $\alpha, \beta$ be distinct numbers in $\left(t, \eta_{1}(t)\right)$, then since $\left(E_{4}\right)$ is self adjoint $w(x) \equiv W\left[u_{3}(x, t), u_{3}(x, \alpha), u_{3}(x, \beta)\right]$ is a nontrivial solution to $\left(E_{4}\right)$ satisfying $y(t)=y(\alpha)=y(\beta)=y\left(\eta_{1}(t)\right)=0$. The remainder of the proof is the same as in Theorem 2.10.

3. Ordering theorems for $r_{i j}(t), z_{i j}(t), r_{i j k}(t), z_{i j k}(t)$, and $r_{1111}(t)$. Theorem 3.1 was proved by Hartman [8], and, more recently, by Opial [11]. We state this theorem here without proof.

THEOREM 3.1. For $t \in[a, \infty), \eta_{1}(t)=r_{1111}(t)$.

The reader should compare Theorem 3.1 to Lemma 2.1 and Theorem 2.10. In particular, if $\eta_{1}(t)<Z_{1}(t)$, then in the definition of $r_{1111}(t)$ we cannot replace the word "infimum" by the word "minimum".

By use of Theorem 3.1 it is fairly easy to prove the next theorem. R. G. Aliev [5] proved the first case of Theorem 3.2 in a somewhat different manner. He also claims that $r_{1111}(t)=\min \left[r_{211}(t), r_{112}(t)\right]$ but his proof is incomplete. However, no counterexample has been produced.

Theorem 3.2. For $t \in[a, \infty)$,

$$
\eta_{1}(t)=\min \left[r_{121}(t), r_{112}(t)\right]=\min \left[r_{121}(t), r_{211}(t)\right] .
$$

Proof. Since $\eta_{1}(t)=r_{1111}(t)$, it suffices to show that

$$
r_{1111}(t) \geqq \min \left[r_{121}(t), r_{112}(t)\right] \quad \text { and } \quad r_{1111}(t) \geqq \min \left[r_{121}(t), r_{211}(t)\right] \text {. }
$$

Let $\rho(t)=\min \left[r_{121}(t), r_{112}(t)\right]$ and assume $r_{1111}(t)<\rho(t)$, then there are points $\alpha, \beta, \gamma$, and $\delta$ such that $t \leqq \alpha<\beta<\gamma<\delta<\rho(t)$ and a nontrivial solution $u(x)$ of $\left(E_{4}\right)$ satisfying $u(\alpha)=u(\beta)=u(\gamma)=u(\delta)=$ 0 and $u^{\prime}(\alpha) u^{\prime}(\beta) u^{\prime}(\gamma)=0$. Since $\delta<\rho(t) \leqq r_{12}(t)$, there is a unique solution $v(x)$ of $\left(E_{4}\right)$ satisfying $v(\alpha)=0, v(\beta)=1, v(\delta)=v^{\prime}(\delta)=0$. Since $\delta<\rho(t) \leqq r_{112}(t), v(x)<0$ for $x \in(\alpha, \delta)$. By Lemma 1.2, there is a nontrivial linear combination of $u(x)$ and $v(x)$ with a double zero in 
$(\beta, \gamma)$, a zero at $\alpha$ and at $\delta$. This contradicts the condition that $\delta<r_{121}(t)$. Similarly $r_{1111}(t) \geqq \min \left[r_{121}(t), r_{211}(t)\right]$.

Several interesting examples illustrate Theorem 3.2. In Example 2.1 Aliev [4] noted that $r_{211}(t)=\infty$, it follows from Theorem 3.2 that $\eta_{1}(t)=z_{121}(t)$. In Example 2.2, $r_{112}(t)=\infty$ and hence $\eta_{1}(t)=z_{121}(t)$. As a third example if in equation $\left(E_{4}\right), q_{1}(x) \leqq 0, i=1,2,3,4$, then $r_{121}(a)=$ $r_{31}(a)=r_{13}(a)=\infty$ and so by Theorem $3.2 \eta_{1}(a)=r_{112}(a)=r_{22}(a)=r_{211}(a)$.

Aliev [4] quotes the first inequality in the next theorem and states that he proved it in a paper [1] unavailable to the author. Theorem 3.3 follows easily from Lemma 1.2 .

Theorem 3.3. If $t \in[a, \infty)$, then

$$
\begin{aligned}
& r_{211}(t) \geqq \min \left[r_{31}(t), r_{22}(t)\right], \\
& r_{112}(t) \geqq \min \left[r_{13}(t), r_{22}(t)\right] .
\end{aligned}
$$

In the next three theorems we consider the cases where either $r_{13}(t)$ or $r_{31}(t)$ is less than $r_{22}(t)$. Note that in Example $2.1 r_{13}(t)<r_{31}(t)=$ $r_{22}(t)=\infty$ and in Example $2.2 r_{31}(t)<r_{13}(t)=r_{22}(t)=\infty$. Also, in the more familiar self adjoint cases, $r_{31}(t)=r_{13}(t)$. In particular, for the differential equation $y^{i v}+y=0$ we have $r_{13}(t)=r_{31}(t)<r_{22}(t)=\infty$, and for the differential equation $y^{i v}-y=0$ we have

$$
r_{22}(t)<r_{13}(t)=r_{31}(t)=\infty \text {. }
$$

THEOREM 3.4. If, for $t \in[a, \infty), r_{13}(t)<r_{22}(t)$, then

$$
r_{13}(t)=r_{112}(t)=z_{112}(t) \text {. }
$$

Proof. By Theorem 3.3, $r_{112}(t) \geqq \min \left[r_{13}(t), r_{22}(t)\right]=r_{13}(t)$. Hence to complete the proof of this theorem it suffices to show that given $\varepsilon>0$, but small enough so that $r_{13}(t)+\varepsilon<r_{22}(t)$, there is a nontrivial solution of $\left(E_{4}\right)$ with a $1-1-2$ distribution of zeros on $\left[t, r_{13}(t)+\varepsilon\right]$ and with a zero at $t$. Since $r_{13}(t)+\varepsilon<r_{22}(t)$ there is a point $\alpha \in\left(t, r_{13}(t)\right)$ such that $r_{13}(\alpha)=z_{13}(\alpha) \in\left(r_{13}(t), r_{13}(t)+\varepsilon\right)$ [4]. Let $\beta=z_{13}(\alpha)$, then there is a nontrivial solution $u(x)$ of $\left(E_{4}\right)$ with a triple zero at $\beta$ and a zero at $\alpha$ where $t<\alpha<\beta<r_{13}(t)+\varepsilon$. If $u(t)=0$, then $r_{13}(t)=$ $r_{112}(t)=z_{112}(t)$. If $u(t) \neq 0$, then let $v(x)$ be a nontrivial solution of $\left(E_{4}\right)$ with a zero at $t$ and a double zero at $\beta$. If $v(x) \neq 0$ for $x \in(t, \beta)$, then by Lemma 1.2 there is a nontrivial linear combination of $u(x)$ and $v(x)$ with a double zero in $(\alpha, \beta)$ and a double zero at $\beta$. This contradicts $\beta<r_{22}(t)$. Therefore $v(x)$ has a zero in $(\alpha, \beta)$ and we have $r_{13}(t)=r_{112}(t)=z_{112}(t)$.

Lemma 3.5 was proved by R. G. Aliev in a paper [3] unavailable 
to the author and is reproved here for the sake of completeness.

LEMmA 3.5. For $t \in[a, \infty), r_{31}(t) \geqq r_{211}(t)$.

Proof. Let $\beta=r_{31}(t)$ and assume $\beta<r_{211}(t)$. There is a point $\alpha \in[t, \beta)$ such that $u_{3}(\beta, \alpha)=0$ and $u_{3}(x, \alpha)>0$ for $x \in(\alpha, \beta)$. If $\beta$ is a double zero of $u_{3}(x, \alpha)$, then $u_{3}(x, \alpha)>0$ for $x \in\left(\beta, r_{211}(t)\right)$. Let $\gamma \in\left(\beta, r_{211}(t)\right)$ and $\mu \in(\alpha, \beta)$ and take $v(x)$ to be the solution of $\left(E_{4}\right)$ with $v(\alpha)=v^{\prime}(\alpha)=0=v(\gamma)$ and $v(\mu)=u_{3}(\mu, \alpha)$. Since $\gamma<r_{211}(t)$, $v(x)>0$ for $x \in(\alpha, \gamma)$. It follows that the difference $u_{3}(x, \alpha)-v(x)$ is a nontrivial solution of $\left(E_{4}\right)$ with a double zero at $\alpha$, a zero at $\mu$, and a zero in $(\beta, \gamma)$. This contradicts the inequality $\gamma<r_{211}(t)$. Therefore $\beta$ is either a simple zero or a triple zero of $u_{3}(x, \alpha)$. In either case $u_{3}(x, \alpha)<0$ for $x \in\left(\beta, r_{211}(t)\right)$. Let $\mu \in(\alpha, \beta)$ and $\nu \in\left(\beta, r_{211}(t)\right)$, then, since $t<\mu<\nu<r_{211}(t)$, there is a unique solution $z(x)$ of $\left(E_{4}\right)$ satisfying $z(\alpha)=z^{\prime}(\alpha)=0, z(\mu)=(1 / 2) u_{3}(\mu, \alpha)$, and $z(\nu)=(1 / 3) u_{3}(\nu, \alpha)$. Since $z(x)$ and $u_{3}(x, \alpha)$ are linearly independent, $D_{2} z(\alpha) \neq 0$, and there are two possibilities. If $D_{2} z(\alpha)>0$, then $u_{3}(x, \alpha)-z(x)$ has a double zero at $\alpha$, a zero in $(\alpha, \mu)$ and a zero in $(\mu, \nu)$. If $D_{2} z(\alpha)<0$, then $z(x)$ has a double zero at $\alpha$, a zero in $(\alpha, \mu)$ and a zero in $(\mu, \nu)$. In both cases we contradict the inequality $\nu<r_{211}(t)$.

TheOREM 3.6. If, for $t \in[a, \infty), r_{31}(t)<r_{22}(t)$, then

$$
r_{31}(t)=r_{211}(t)=z_{211}(t) \text {. }
$$

Proof. It follows from Theorem 3.3 and Lemma 3.5 that $r_{31}(t)=$ $r_{211}(t)$. To show that $r_{31}(t)=z_{211}(t)$ it suffices to show that given $\varepsilon>0$, but small enough so that $r_{31}(t)+\varepsilon<r_{22}(t)$, that there is a nontrivial solution of $\left(E_{4}\right)$ with a 2-1-1 distribution of zeros on $\left[t, r_{13}(t)+\varepsilon\right]$ with at least a double zero at $t$. Since $r_{31}(t)+\varepsilon<r_{22}(t), r_{31}(t)=z_{31}(t)$ and hence $u_{3}\left(r_{31}(t), t\right)=0$ [4]. The number $\varepsilon$ can be taken so that $u_{3}(x, t) \neq 0$ for $x \in\left(r_{31}(t), r_{31}(t)+\varepsilon\right)$. Let $\alpha \in\left(r_{31}(t), r_{31}(t)+\varepsilon\right)$ and let $v(x)$ be a nontrivial solution of $\left(E_{4}\right)$ with $v(t)=v^{\prime}(t)=0, v(\alpha)=0$. If $v(x) \neq 0$ for $x \in(t, \alpha)$, then if we apply Lemma 1.2 to $v(x)$ and $u_{3}(x, t)$ we contradict the inequality $r_{31}(t)<r_{22}(t)$. Hence $v(x)$ has a zero in $\left(t, r_{31}(t)\right)$ and so $r_{31}(t)=z_{211}(t)$.

THEOREM 3.7. If, for $t \in[a, \infty), r_{13}(t)=r_{31}(t)<r_{22}(t)$, then

$$
r_{31}(t)=r_{13}(t)=r_{121}(t)=z_{121}(t) \text {. }
$$

Proof. Since $\eta_{1}(t)=\min \left[r_{13}(t), r_{31}(t), r_{22}(t)\right]=r_{31}(t)$, it suffices to show that given $\varepsilon>0$, but small enough so that $\eta_{1}(t)+\varepsilon<r_{22}(t)$, 
$u_{3}(x, t)<0$ for $x \in\left(\eta_{1}(t), \eta_{1}(t)+\varepsilon\right)$, and $u_{3}\left(x, \eta_{1}(t)>0\right.$ for $x \in\left(\eta_{1}(t), \eta_{1}(t)+\varepsilon\right)$, that there is a nontrivial solution of $\left(E_{4}\right)$ with a $1-2-1$ distribution of zeros on $\left[t, \eta_{1}(t)+\varepsilon\right]$ with a zero at $t$. Let $v(x)$ be a nontrivial solution of $\left(E_{4}\right)$ with a double zero at $t$ and a zero in $\left(\eta_{1}(t), \eta_{1}(t)+\varepsilon\right)$. If $v\left(\eta_{1}(t)\right)=0$, then it is easy to see that there would be a nontrivial linear combination of $u_{3}(x, t)$ and $v(x)$ with a double zero at $t$ and at $\eta_{1}(t)$. This contradicts $\eta_{1}(t)<r_{22}(t)$ and so $v\left(\eta_{1}(t)\right) \neq 0$. If $v(x) \neq 0$ for $x \in\left(t, \eta_{1}(t)\right)$, then if we apply Lemma 1.2 to $u_{3}(x, t)$ and $v(x)$ we contradict the inequality $\eta_{1}(t)<r_{22}(t)$. Hence $v(x)$ has a zero (and only one, call it $\alpha)$ in $\left(t, \eta_{1}(t)\right)$. Let $\beta$ be the first zero of $v(x)$ in $\left(\eta_{1}(t)\right.$, $\left.\eta_{1}(t)+\varepsilon\right)$. It follows by Lemma 1.2 that there is a nontrivial linear combination of $u(x)$ and $v(x)$ with a zero at $t$, a double zero in $(t, \alpha)$ and a zero in $\left(\eta_{1}(t), \beta\right)$.

Theorem 3.8. If $r_{22}(t)<\min \left[r_{31}(t), r_{13}(t)\right]$, then

$$
r_{22}(t)=r_{211}(t)=z_{211}(t)=r_{112}(t) \text {. }
$$

Proof. Let $\rho(t) \equiv \min \left[r_{31}(t), r_{13}(t)\right]$ and let $u(x)$ be a nontrivial solution of $\left(E_{4}\right)$ with exactly a double zero at $t$ and a double zero at $\eta_{1}(t)$. By Lemma $2.4 u(x)$ does not have a zero in $\left(t, \eta_{1}(t)\right)$. It is easy to see that there is a nontrivial linear combination of $u_{3}(x, t)$ and $u(x)$ in $\left(t, \eta_{1}(t)\right)$, and a zero at $p$ where $p<\eta_{1}(t)+\varepsilon<\rho(t)$. Hence $r_{22}(t)=$ $z_{211}(t)$.

To show $\eta_{1}(t)=r_{112}(t)$ it suffices to show that for $\varepsilon<0$, but small enough so that $\eta_{1}(t)+\varepsilon<\rho(t)$, there is a nontrivial solution of $\left(E_{4}\right)$ with a 1-1-2 distribution of zeros on $\left[t, \eta_{1}(t)+\varepsilon\right]$. Let $\delta \in\left(t, \eta_{1}(t)\right)$ such that $\eta_{1}(\delta) \in\left(\eta_{1}(t), \eta_{1}(t)+\varepsilon\right)$. Since $\eta_{1}(t)+\varepsilon<\rho(t), \eta_{1}(\delta)=r_{22}(\delta)$. Let $w(x)$ be a nontrivial solution of $\left(E_{4}\right)$ with a double zero at $\delta$ and a double zero at $r_{22}(\delta)$. If $u(x)$ has a zero in $(t, \delta)$, then $\eta_{1}(t)=r_{112}(t)$. If $u(x)$ does not have a zero in $(t, \delta)$, then there is a nontrivial linear combination of $u(x)$ and $v(x)$ with a zero in $(t, \delta)$, a zero in $\left(\delta, r_{22}(\delta)\right)$ and a double zero at $r_{22}(\delta)<\eta_{1}(t)+\varepsilon$.

For the equation $y^{i v}+y^{\prime \prime}=0, \eta_{1}(t)=r_{22}(t)=t+2 \pi$ and $r_{31}(t)=$ $r_{13}(t)=\infty$. It follows from Theorem 3.8 that $z_{211}(t)=r_{112}(t)=t+2 \pi=$ $z_{112}(t)$.

Corollary 3.9. Let $\left(E_{4}\right)$ be self adjoint.

(i ) If $\eta_{1}(t)<r_{22}(t)$, then $\eta_{1}(t)=z_{211}(t)=z_{121}(t)=z_{122}(t)$.

(ii) If $\eta_{1}(t)<r_{31}(t)$, then $\eta_{1}(t)=z_{211}(t)=r_{112}(t)$.

Proof. Corollary 3.9 follows directly from Theorems 3.4, 3.6, 3.7, and 3.8 .

One notices the absence of $r_{121}(t)$ in part (ii) of Corollary 3.9. For 
the equations of the form $\left(r y^{\prime \prime}\right)^{\prime \prime}+p y=0$ where $r(x)>0, p(x) \leqq 0$, $x \in[a, \infty), r(x), p(x) \in C[a, \infty)$ for which $\eta_{1}(t)=r_{22}(t)<\infty$ [6] the hypothesis of part (ii) of Corollary 3.9 is fulfilled but $\eta_{1}(t)<r_{31}(t)=r_{13}(t)=$ $r_{121}(t)=\infty[10]$.

The author wishes to thank Professor J. H. Barrett for his advice and assistance.

\section{REFERENCES}

1. R. G. Aliev, On the guestion of distribution of zeros of solutions of linear differential equations of fourth order, (Russian), Rechts. of Scientific Aspirants Conference for 1962, Kazan Institute, 1962.

2. On the question of concrete criteria for the estimation of the lengths of precritical intervals, (Russian), Izv. Vyss. Ucebn. Zaved. Matematika (41) 5 (1964), 3-7.

3. L Laws of the distributions of zeros of solutions of linear equations of fourth order, (Russian), Sb. Aspirantsh, Kazan Institute, 1964, 15-30.

4. - On the sign of the Green's function for a boundary-value problem for a fourth order differential equation, (Russian), Izv. Vyss. Ucebn. Zaved. Matematika (43) 6 (1964), 3-9.

5. - On certain properties of solutions of ordinary differential equations of fourth order, (Russian), Izv. Vyss. Ucebn. Zaved. Matematika (45) 2 (1965), 3-6.

6. J. H. Barrett, Oscillation theory of ordinary linear differential equations, Associated Western Universities Differential Equations Symposium, Boulder, Colorado, 1967.

7. J. M. Dolan, Oscillatory behavior of solutions of linear differential equations of third order, Unpublished doctoral dissertation, The University of Tennessee, Knoxville, Tennessee, 1967.

8. P. Hartman, Unrestricted n-parameter families, Rend. Circ. Mat. Palermo (2) 7 (1958), 123-142.

9. D. B. Hinton, Disconjugate properties of a system of differential equations, J. Differential Equations 2 (1966), 420-437.

10. W. Leighton and Z. Nehari, On the oscillation of solutions of self-adjoint linear differential equations of the fourth order, Trans. Amer. Math. Soc. 89 (1958), 325-377. 11. Z. Opial, On a theorem of O. Arama, J. of Differential Equations 3 (1967), 38-91. 12. T. L. Sherman, Porperties of solutions of $N^{\text {th }}$ order linear differential equations, Pacific J. Math. 15 (1965), 1045-1060.

Received August 28, 1968, and in revised form January 13, 1969. This paper is part of the author's dissertation.

This work was supported, in part, by NASA Grant Number NGR 43-001-029.

The University of Tennessee

AND

The University of Nebraska 


\section{PACIFIC JOURNAL OF MATHEMATICS}

\section{EDITORS}

H. ROYDEN

Stanford University

Stanford, California

\section{RichaRd PIERCE}

University of Washington Seattle, Washington 98105
J. DugundJI

Department of Mathematics

University of Southern California

Los Angeles, California 90007

BASIL GORDON

University of California

Los Angeles, California 90024

\section{ASSOCIATE EDITORS}
E. F. BECKENBACH
B. H. NeUMANN
F. WOLF
K. YOSHIDA

\section{SUPPORTING INSTITUTIONS}

UNIVERSITY OF BRITISH COLUMBIA CALIFORNIA INSTITUTE OF TECHNOLOGY

UNIVERSITY OF CALIFORNIA

MONTANA STATE UNIVERSITY

UNIVERSITY OF NEVADA

NEW MEXICO STATE UNIVERSITY

OREGON STATE UNIVERSITY

UNIVERSITY OF OREGON

OSAKA UNIVERSITY

UNIVERSITY OF SOUTHERN CALIFORNIA

\author{
STANFORD UNIVERSITY \\ UNIVERSITY OF TOKYO \\ UNIVERSITY OF UTAH \\ WASHINGTON STATE UNIVERSITY \\ UNIVERSITY OF WASHINGTON \\ AMERICAN MATHEMATICAL SOCIETY \\ CHEVRON RESEARCH CORPORATION \\ TRW SYSTEMS \\ NAVAL WEAPONS CENTER
}

The Supporting Institutions listed above contribute to the cost of publication of this Journal, but they are not owners or publishers and have no responsibility for its content or policies.

Mathematical papers intended for publication in the Pacific Journal of Mathematics should be in typed form or offset-reproduced, double spaced with large margins. Underline Greek letters in red, German in green, and script in blue. The first paragraph or two must be capable of being used separately as a synopsis of the entire paper. It should not contain references to the bibliography. Manuscripts, in duplicate if possible, may be sent to any one of the four editors. Please classify according to the scheme of Math. Rev. 36, 1539-1546. All other communications to the editors should be addressed to the managing editor, Richard Arens, University of California, Los Angeles, California, 90024.

50 reprints are provided free for each article; additional copies may be obtained at cost in multiples of 50 .

The Pacific Journal of Mathematics is published monthly. Effective with Volume 16 the price per volume (3 numbers) is $\$ 8.00$; single issues, $\$ 3.00$. Special price for current issues to individual faculty members of supporting institutions and to individual members of the American Mathematical Society: $\$ 4.00$ per volume; single issues $\$ 1.50$. Back numbers are available.

Subscriptions, orders for back numbers, and changes of address should be sent to Pacific Journal of Mathematics, 103 Highland Boulevard, Berkeley, California, 94708.

PUBLISHED BY PACIFIC JOURNAL OF MATHEMATICS, A NON-PROFIT CORPORATION

Printed at Kokusai Bunken Insatsusha (International Academic Printing Co., Ltd.), 7-17. Fujimi 2-chome, Chiyoda-ku, Tokyo, Japan. 


\section{Pacific Journal of Mathematics}

\section{Vol. 30, No. $3 \quad$ November, 1969}

Willard Ellis Baxter, Topological rings with property $(Y) \ldots \ldots \ldots \ldots . \ldots 5$

Sterling K. Berberian, Note on some spectral inequalities of $C . R$.

Putnam ..................................... 573

David Theodore Brown, Galois theory for Banach algebras . ........... 577

Dennis K. Burke and R. A. Stoltenberg, A note on p-spaces and Moore spaces ........................................ 601

Rafael Van Severen Chacon and Stephen Allan McGrath, Estimates of positive contractions....................................... 609

Rene Felix Dennemeyer, Conjugate surfaces for multiple integral problems in the calculus of variations ........................... 621

Edwin O. Elliott, Measures on countable product spaces.............. 639

John Moss Grover, Covering groups of groups of Lie type .............. 645

Charles Lemuel Hagopian, Concerning semi-local-connectedness and cutting in nonlocally connected continua .................. 657

Velmer B. Headley, A monotonicity principle for eigenvalues ........... 663

John Joseph Hutchinson, Intrinsic extensions of rings . . . . . . . . . . . . . 669

Harold H. Johnson, Determination of hyperbolicity by partial

prolongations .................................. 679

Tilla Weinstein, Holomorphic quadratic differentials on surfaces in $E^{3} \ldots 697$

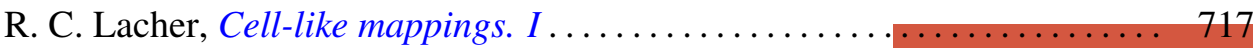

Roger McCann, A classification of centers

Curtis L. Outlaw, Mean value iteration of nonexpansive mappings in a

Banach space...

Allan C. Peterson, Distribution of zeros of solutions of a fourth order

differential equation.

Bhalchandra B. Phadke, Polyhedron inequality and strict convexity .. 765 Jack Wyndall Rogers Jr., On universal tree-like continua .

Edgar Andrews Rutter, Two characterizations of quasi-Frobenius rings

G. Sankaranarayanan and C. Suyambulingom, Some renewal theorems concerning a sequence of correlated random variables...

Joel E. Schneider, A note on the theory of primes........ . .

Richard Peter Stanley, Zero square rings .................

Edward D. Tymchatyn, The 2-cell as a partially ordered space

Craig A. Wood, On general Z.P.I.-rings................ 